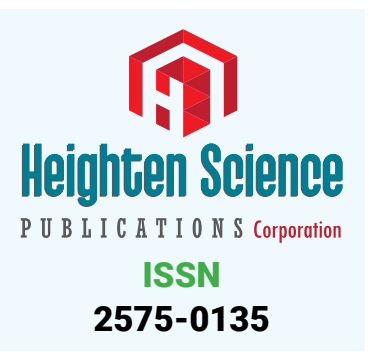

*Address for Correspondence: Charles $\mathrm{AO}$ Midega, International Centre of Insect Physiology and Ecology (ICIPE), P.O. Box, 30772-00100, Nairobi, Kenya, Tel: +254 59 22216/7/8; Email: cmidega@icipe.org

Submitted: 08 August 2018

Approved: 28 August 2018

Published: 29 August 2018

Copyright: @ 2018 Midega CAO, et al. This is an open access article distributed under the Creative Commons Attribution License, which permits unrestricted use, distribution, and reproduction in any medium, provided the original work is properly cited.

Keywords: Armored scale insect; Japanese silver tree; Life history; Natural enemies

\section{Life history strategies of the armored scale, Aulacaspis alisiana (Hemiptera: Coccoidea: Diaspididae) on the Japanese silver tree Neolitsea sericea (BI.) Koidz. (Lauraceae) in Fukuoka, Japan}

\author{
Charles AO Midega ${ }^{1 *}$ and Keiji Takasu ${ }^{2}$ \\ ${ }^{1}$ International Centre of Insect Physiology and Ecology (ICIPE), P.O. Box, 30772- 00100, Nairobi, \\ Kenya \\ 2Department of Bioresource Sciences, Faculty of Agriculture, Kyushu University, 6-10-1, \\ Hakozaki, Higashi-Ku, Fukuoka City, 812-8581, Japan
}

\section{Abstract}

The armored scale Aulacaspis alisiana, is a serious invasive pest of the Japanese silver tree, Neolitsea sericea, causing serious damage to the tree in Japan. However there are currently no control approaches available for it, complicated by shortage of information on the pest. We studied life history strategies of $A$. alisiana on $N$. sericea in Fukuoka Prefecture with a view to providing a basis for formulating sustainable control based on an understanding of the behavior of the pest and potential role of its natural enemies. We established that $A$. alisiana had three overlapped generations in Fukuoka, with generation times ranging between 65 and 71 days. The adults were relatively fecund, with each female producing between 60 and 67 eggs, with high hatchability, $>78 \%$. The pest settled on the lower side of leaves, and although it generally preferred younger leaves, it did not attack newly emerged leaves. Natural enemy groups comprising ants, spiders and beetles (coccinelids) played an important role in regulation of the pest population, with natural mortality of about $30 \%$. They could thus form a critical component of an integrated management approach for the pest in Fukuoka.

\section{Introduction}

The Japanese silver tree, Neolitsea sericea (Bl.) Koidz. (Lauraceae), is an important multi-purpose tree in the Asian continent, distributed through southern China and Japan (Honshu, Shikoku, Kyushu, and Ryukyu Islands), and occurs in a few localities in the southern coastal area of the Korean Peninsula [1,2]. In China, where it is listed as an endangered tree species [1], it is historically associated with Buddhism, and is referred to as the 'Buddhism tree'. Because of its graceful shape and religious connotation, it is commonly used as an ornamental tree in public gardens and streets [2]. A concoction of its leaf oil contains a knock-down effect on some house dust and plant mites [3]. Like most other trees, it affords wildlife refuge and people benefit from it through an increase in property value and wood products that are produced, such as utensils, buildings and fuels. The seeds produce burning oil and wax of candle.

Continued presence and use of $N$. sericea is endangered by the armored scale, Aulacaspis alisiana (Homiptera: Coccoidea: Diaspididae). This insect is host-specific 
to $N$. sericea in Japan. The cryptic size ( 0.5 to $1.5 \mathrm{~mm}$ in length) and shape (flattened bodies) of armored scales often allows populations to be undetected until economically damaging levels are reached [4]. Scales directly affect plant growth by their feeding, which involves penetration of their stylets into the phloem and uptake of sap as food [5]. This results in branch dieback, reduced tree growth rates, and sooty mould, which grow on honeydew secreted by the scales.

In spite of the damage and danger posed by A. alisiana to $N$. sericea (Figure 1), information is unavailable on life history of the pest on its host. We therefore studied life history strategies of $A$. alisiana on $N$. sericea with a view to providing a basis for formulating sustainable control based on an understanding of the behavior of the pest and potential role of its natural enemies.

\section{Materials and Methods}

\section{Study sites}

These studies were conducted in two natural forests, Ino $\left(33^{\circ}-39^{\prime} \mathrm{N}, 130^{\circ} 31^{\prime} \mathrm{E}\right)$ and

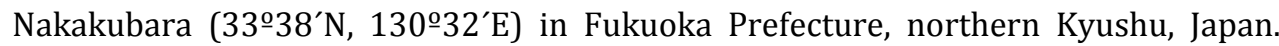
These two forests are characterized by a large number of tree species, including the famous Japanese cedar, Cyrptomeria japonica, also known as Sugi. Additionally, there are a number of trees in the laurel family (Lauraceae), evergreen members of the beech family (Fagaceae), as well as the tea family (Theaceae).

\section{Fecundity}

To estimate fecundity of A. alisiana, a methodology adapted from Ozaki et al. [6], was used with modifications. Twenty trees were identified at both sites and two infested leaves randomly marked from each tree and assigned numbers. The number of female A. alisiana were then counted from each leaf and recorded. Because A. alisiana deposits eggs under its scales, we counted the number of nymphs (crawlers) that emerged from female scales twice a week for two weeks. After the last observation, the numbers of eggs and crawlers that remained under the female scales were counted. Fecundity was estimated by summing all these numbers and dividing by the number of female scales on each leaf.

\section{Life cycle and generation time of A. alisiana}

We randomly assigned 10 trees at each site, from which two leaves were randomly selected on each tree. The number of eggs and emerged crawlers were then counted, recorded and their positions marked on the opposite side of the leaves. These, together with any other emerged crawlers on the leaves were observed twice a week until they became adults. The average time taken to develop to adult stage was calculated to estimate the generation time. This was conducted over three generations of the insect at both sites.

\section{Settling behavior of $A$. alisiana}

Because it had been observed that A. alisiana had more than one generation in a year in Fukuoka (J. Yukawa, Pers. communication), and N. sericea trees extended their new

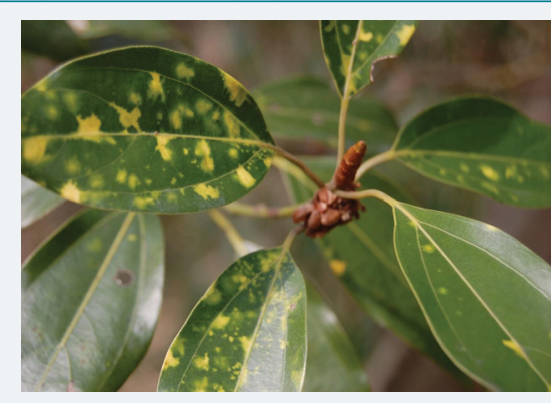

Figure 1: Leaves of Neolitsea sericea showing damage (discoloration) caused by Aulacaspis alisiana. 
leaves only once a year (during spring, from the month of March), it was thought that the first generation of the insect might use the new leaves while the second and third generations use the only available old leaves. Thus we monitored preference of the insect life stages for particular leaves (age and size). We randomly selected ten trees at Ino forest from which ten infested leaves were picked per tree. We then observed the leaves for A. alisiana life stages and determined the sizes of the leaves and their ages in years, based on estimation of the time when they emerged.

\section{Natural mortality of $A$. alisiana}

The role of natural enemies on natural regulation of $A$. alisiana populations at Ino forest was assessed through exclusion studies using modifications of methodologies of [7]. Twenty infested tree branches were selected and number of crawlers on each branch counted: ten were randomly assigned to 'treatment' while a similar number was assigned to 'control'. 'Treatment' branches had natural enemies excluded by covering infested leaves with fine mosquito netting material, with a band of insect trap adhesive applied at the base of the leaves to keep away crawling predators. The 'control' branches were left exposed. Population dynamics of $A$. alisiana on the two sets of leaves was assessed on a weekly basis. Any predators encountered on the leaves were counted according to their morphospecific groups. The insect's life stages collected from the trees (dead and alive) were maintained in the laboratory and examined for any parasitoid emergence.

\section{Data analysis}

Data were averaged per tree, branch or leaf and subjected to either one-way analysis of variance or two-sample t-test. Data were analyzed using R-software [8], and mean separation done using Tukey HSD test where necessary.

\section{Results and Discussion}

\section{Fecundity}

Each A. alisiana female oviposited on average 59.8 eggs, out of which $78.8 \%$ hatched at Ino, and 67.4 eggs, out of which $84.6 \%$ hatched at Nakakubara. The number of eggs oviposited by A. alisiana, and proportions that hatched, did not significantly differ between the two sites (eggs oviposited: $\mathrm{t}=-1.6$; $\mathrm{P}=0.011$; proportions of eggs hatched: $\mathrm{t}=-1.9 ; \mathrm{P}=0.04)$.

\section{Life cycle and generation time of $A$. alisiana}

A. alisiana has three overlapped generations in Fukuoka, with generation time ranging between 65 and 71 days. The first generation emerges in mid-April, extending through to late July. The second generation emerges from late June through to early September, while the third and last generation nymphs begin to emerge in late August and extends through the rest of the year, over-wintering as adults and mature nymphs. Generation times did not significantly differ within and between sites. At Ino, mean generation times were 71.2(0.9), 70.4(0.7) and 68.7(1.3) days for generations 1,2 and 3 , respectively. There were no significant differences between these times $\left(\mathrm{F}_{2,27}=1.8\right.$; $\mathrm{p}=0.183)$. Similarly, mean generation times were 68.4(1.5), 67.6(1.8) and 65.4(1.6) days for generations 1,2 and 3 , respectively, with no significant differences between the times $\left(\mathrm{F}_{2,27}=0.86 ; \mathrm{p}=0.435\right)$.

\section{Settling behaviour}

A. alisiana settles on the lower side of the leaves. The number of $A$. alisiana life stages encountered did not significantly differ among leaves of different ages $\left(F_{2,27}=0.21\right.$; $\mathrm{p}=0.813)$ and sizes $\left(\mathrm{F}_{2,27}=0.07 ; \mathrm{p}=0.932\right)$. Insects averaged 244.4(49.3), 218.8(34.4) and 199.4(61.2) per leaf one year, two years and over 2 years of age, respectively. With regards to leaf size, insects averaged 396.5(51.0), 383.5(42.5) and 410.8(59.7) 
per small $\left(0-50 \mathrm{~cm}^{2}\right)$, medium $\left(51-100 \mathrm{~cm}^{2}\right)$ and large $\left(>100 \mathrm{~cm}^{2}\right)$ leaves, respectively. These results suggest that the insect does not display any preference for leaf sizes and ages. However, there was a progressive decrease in the number of A. alisiana with leaf age. In addition, while adults of this insect have been reported to display a parallel alignment (males and females) on different lines, often along leaf veins or leaf edges (J. Yukawa, Pers. Communication), we observed that their distribution on $N$. sericeae leaves was more or less random. Similarly, no insects were observed on the newly emerged leaves, implying there is no synchronization between the first generation and emergence of new leaves.

\section{Natural mortality of $A$. alisiana}

The potential generalist natural enemy guild comprised mainly spiders, ants and beetles (coccinelids) in the order of abundance, with an average of 28.6 spiders, 19.8 ants and 7.5 beetles per tree. No parasitoids were observed. Disappearance, attributable to predation, of $A$. alisiana nymphs was significantly higher on control than on exclusion branches $(\mathrm{t}=3.96, \mathrm{df}=18 ; \mathrm{p}<0.001)$. Mean disappearance rates were $29.7(5.1) \%$ and $6.2(2.7) \%$ on control and exclusion branches, respectively. These results indicate an important contribution of predators in natural regulation of A. alisiana population in these sites. Subsequent (data not provided) showed that mortality progressively increased from the medium to large nymphs, with mortality at the small nymph stage being significantly lower. This implies inadequate nutritional resource as nymphs mature and their nutritional needs increase.

In conclusion, $A$. alisiana has three overlapped generations in Fukuoka, with generation times ranging between 65 and 71 days. The adults are relatively fecund, with each female producing between 60 and 67 eggs, with high hatchability, $>78 \%$. The pest settles on the lower side of leaves, and although it generally prefers younger leaves, it does not seem to attack newly emerged leaves. Natural enemies comprising ants, spiders and beetles (coccinelids) play an important role in pest regulation, with natural mortality being about $30 \%$. They could thus form a critical component of an integrated management approach for the pest in Fukuoka.

\section{Acknowledgement}

The authors greatly acknowledge the financial support received from the Japan Society for the Promotion of Science.

\section{References}

1. Fu LG. Chinese plant red book. Beijing: Science Press. 1992.

2. Wang ZS, An SQ, Liu H, Leng X, Zheng JW, et al. Genetic structure of the endangered plant Neolitsea sericea (Lauraceae) from the Zhoushan Archipelago using RAPD markers. Ann Bot. 2005; 95: 305313. Ref.: https://tinyurl.com/y9zenhsu

3. Furuno $T$, Terada $Y$, Yano $S$, Uugara $T$, Jodai $S$. Activities of leaf oils and their components from Lauraceae trees against house dust mites. Mokuzai Gakkaishi. 1994; 40: 78-87. Ref.: https://tinyurl.com/ya74f8wv

4. Hodges G, Howard FW, Buss EA. Pest Alert: Update on management methods for cycad Aulacaspis scale. 2005. Ref.: https://tinyurl.com/y9au4rah

5. Vranjic JA. Effects on Host Plant. World Crop Pests. 1997; 7: 323-336. Ref.: https://tinyurl.com/ya9f945n

6. Ozaki K, Kitamura S, Subiandoro E, Taketani A. Life history of Aulacaspis marina Takagi and Williams (Hom., Coccoidea), a new pest of mangrove plantations in Indonesia, and its damage to mangrove seedlings. J Appl Entomol. 1999; 123: 281-284. Ref.: https://tinyurl.com/ycomxsrq

7. Midega CAO, Khan ZR, Van den Berg J, Ogol CKPO, Pickett JA, et al. Maize stemborer predator activity under 'push-pull' system and Bt-maize: a potential component in managing Bt resistance. Int J Pest Manage. 2006; 52: 1-10. Ref.: https://tinyurl.com/ydcu65er

8. R Core Team. R: A language and environment for statistical computing. R Foundation for Statistical Computing, Vienna, Austria. 2014. Ref.: https://tinyurl.com/nksjxfx 